\title{
INVESTIGACIONES
}

\section{Valoración del trabajo colaborativo entre profesores de escuelas básicas de Tomé, Chile}

\author{
Colaborative Work's Valuation among Teachers \\ of Primary Schools of Tomé, Chile
}

\author{
Valoração do trabalho colaborativo entre professores \\ de escolas de ensino fundamental de Tomé, Chile
}

\author{
Felipe Francisco Rodríguez Rojas, ${ }^{a}$ Carlos Javier Ossa Cornejo. ${ }^{b}$

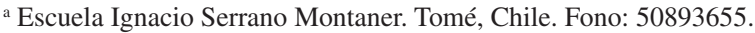 \\ Correo electrónico: ferodriguez85@gmail.com

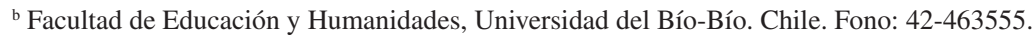 \\ Correo electrónico: cossa@ubiobio.cl
}

\begin{abstract}
RESUMEN
Este artículo presenta un estudio exploratorio de naturaleza cualitativa realizado en la comuna de Tomé, Chile. El propósito fue develar la percepción sobre el trabajo colaborativo que tienen los profesores de educación regular y educación especial, en el contexto de Programas de Integración Escolar. Se realizaron entrevistas semiestructuradas, las que se analizaron con el método de análisis sociológico. Los resultados señalan la existencia de algunos grados de colaboración entre los docentes, apreciándose la influencia de concepciones tradicionales y discriminadoras entre las modalidades de educación regular y educación especial según el discurso de los participantes. Se observa que el trabajo colaborativo se ve dificultado por un problema de cohesión social en la cultura y la organización escolar.
\end{abstract}

Palabras clave: trabajo colaborativo, co-enseñanza, educación inclusiva, programas de integración escolar, gestión curricular.

\section{ABSTRACT}

This paper is about an exploratory and qualitative study done in Tomé, Chile. The purpose is to reveal perceptions about collaborative work of regular and special education's teachers, in the context of Educational Integration Programs. Semi-structured interviews were conducted, which were analyzed through method of sociological analysis. The results of the analysis of interviews show certain levels of collaboration between teachers, and traditional and discriminatory conceptions of special and regular education were likewise found. Also, the collaborative work seems complicated due to a social cohesion problem in the school's culture and organization.

Key words: collaborative work, co-teaching, inclusive education, educational integration programs, curriculum management.

\section{RESUMO}

Apresenta-se um estudo exploratório, de natureza qualitativa, realizado na comunidade de Tomé, Chile. Objetivou-se desvelar a percepção a respeito do trabalho colaborativo que professores do ensino regular e ensino especial têm, no contexto dos Programas de Integração Escolar. Realizaram-se entrevistas semiestruturadas, as quais foram analisadas a partir do método de análise sociológico. Resultados indicam a existência de alguns graus de colaboração entre os docentes, apreciando-se a influência de concepções tradicionais e discriminadoras entre as modalidades de educação regular e educação especial, segundo o discurso dos participantes. Observa-se que o 
trabalho colaborativo apresenta dificuldades por causa de um problema de coesão social na cultura e organização escolar.

Palavras chave: trabalho colaborativo, co-ensino, educação inclusiva, programas de integração escolar, gerenciamento curricular.

\section{INTRODUCCIÓN}

El trabajo colaborativo es una de las principales estrategias organizacionales y curriculares utilizadas para el aprendizaje desde un enfoque inclusivo (Graden y Bauer, 1999; Moliner, 2008; Stainback y Stainback, 1999). Existe mucha evidencia sobre los beneficios del trabajo colaborativo para desarrollar una educación inclusiva, mejorar la calidad de los aprendizajes y favorecer la cooperación entre los profesores (Cramer et al., 2010; Moliner, 2008; Stuart et al., 2006; Villa, Thousand \& Nevin, 2008).

El Ministerio de Educación de Chile entiende el concepto de trabajo colaborativo como los diversos apoyos otorgados por equipos interdisciplinarios a los estudiantes, ya sean dentro del aula como fuera de ella (MINEDUC, 2012a, 2012b, 2009, 2008), centrándose en el trabajo que realizan los equipos de aula para el aprendizaje y participación de todos los estudiantes, especialmente entre los profesores de educación regular y de educación especial (MINEDUC, 2010), de acuerdo a las orientaciones del Decreto 170 de 2009.

Este decreto estableció la obligatoriedad de otorgar horas a los profesores para realizar trabajo colaborativo bajo el modelo de co-enseñanza, en los establecimientos educacionales con Programas de Integración Escolar. Estos programas son una estrategia para fomentar el aprendizaje y la participación de todos los estudiantes en los establecimientos de educación regular, especialmente de aquellos que presentan necesidades educativas especiales (MINEDUC, 2012b). Esto se enlaza con la disposición del Decreto 170 de 2009 que obliga a los profesionales de la educación especial a entregar apoyos en la sala de clases regular, al menos durante 8 horas pedagógicas semanales por curso en los establecimientos con Jornada Escolar Completa Diurna, y 6 horas en aquellos sin Jornada Escolar Completa Diurna, practicando la co-enseñanza durante ese período. Esto implica grandes cambios en la forma de trabajar de los docentes y en la gestión del currículum, los cuales requieren tanto un soporte organizacional como transformaciones en la cultura escolar.

En Chile, las investigaciones realizadas antes de la implementación del Decreto 170 de 2009 señalan una escasa práctica de trabajo colaborativo, tanto entre los profesores como con los profesionales asistentes de la educación, evidenciándose dificultades para integrar los conocimientos profesionales, falta de tiempo, desarrollo de relaciones profesionales asimétricas, resistencia para solicitar y recibir colaboración, poca claridad de roles y focalización en algunos momentos del proceso educativo (Araneda et al., 2008; Castro y Figueroa, 2006).

Una de las formas de trabajo colaborativo que se ha intentado implementar a partir del decreto 170 de 2009 es la co-enseñanza. Esta modalidad ha sido definida como dos o más personas que comparten la responsabilidad de enseñar a un grupo de estudiantes (Cramer et al., 2010; Villa, Thousand \& Nevin, 2008). Si bien involucra principalmente a profesores de educación regular y especial, trabajando, otorgando ayuda y prestando servicios de forma colaborativa para las necesidades de los estudiantes (Cramer et al., 2010), puede ser practicada con otros profesionales. 
La co-enseñanza es una modalidad de trabajo colaborativo especialmente pensada en la lógica de la gestión curricular y la didáctica, pues se desarrolla mediante un proceso de planificación, instrucción y evaluación colaborativa (Murawski, 2008). Esto requiere la combinación de competencias de enseñanza entre los docentes, donde el profesor regular aporta conocimientos curriculares, mientras el profesor de educación especial aporta conocimientos metodológicos (Beamish, Bryer \& Davies, 2006). También implica coordinar el trabajo hacia metas comunes, compartir un sistema de creencias, demostrar paridad en los roles, utilizar un liderazgo distributivo y actuar en forma cooperativa (Villa, Thousand \& Nevin, 2008).

Aunque existen varias formas de practicar la co-enseñanza, lo importante es que los docentes determinen las metas, contenidos, tareas y necesidades de la clase, seleccionando el enfoque de co-enseñanza más apropiado para cada situación (Hughes \& Murawski, 2001). De hecho, en el desarrollo de una unidad curricular se pueden utilizar varios enfoques según los requerimientos particulares de cada clase. Al respecto, los principales enfoques de co-enseñanza son:

1. Co-enseñanza de observación, donde un profesor dirige la totalidad de la clase mientras el otro recoge información académica, conductual y social del grupo clase o de algunos estudiantes en particular.

2. Co-enseñanza de apoyo, consistente en que un profesor toma el rol de conducir la clase mientras el otro se rota entre los estudiantes, proveyéndoles apoyo individual, supervisando, recogiendo información y manejando la conducta.

3. Co-enseñanza en grupos simultáneos, en que los educadores dividen la clase en dos grupos, a los cuales enseñan en forma paralela.

4. Co-enseñanza de rotación entre grupos, en donde los profesores trabajan con grupos diferentes de estudiantes. Los docentes se rotan entre los grupos y también puede existir un grupo que trabaje sin profesor.

5. Co-enseñanza en estaciones, en la cual los docentes dividen el material y los estudiantes en estaciones que funcionan en forma simultánea. Durante el desarrollo de la clase los estudiantes van rotando entre las estaciones. También puede existir una estación que trabaje sin profesor.

6. Co-enseñanza alternativa, que ocurre cuando un docente trabaja con un grupo pequeño de estudiantes desarrollando actividades remediales, de preparación, enriquecimiento y evaluación, mientras el otro docente trabaja con la clase completa.

7. Co-enseñanza complementaria, donde un profesor realiza acciones para mejorar o complementar la enseñanza provista por el otro profesor.

8. Co-enseñanza en equipo, consistente en que los co-educadores desarrollan simultáneamente la clase, alternándose los roles de conducir y apoyar la clase (Friend et al., 2010; Hughes \& Murawski, 2001; Villa, Thousand \& Nevin, 2008).

Si bien estas diferentes maneras de implementar la co-enseñanza pueden utilizarse de forma complementaria, es necesario destacar que la determinación de una u otra modalidad responde a una discusión profesional entre los co-enseñantes, lo cual releva la característica dialógica que presenta como base esta estrategia, siendo por ello un mecanismo participativo y necesario para la efectividad de la práctica docente. 


\section{MÉTODO}

El estudio se enmarcó dentro de un enfoque cualitativo de investigación, de tipo exploratorio, con un diseño de casos múltiples y enmarcado desde la perspectiva hermenéutica, ya que para develar las percepciones de los docentes se decidió interpretar comprensivamente el discurso hablado de los sujetos (Cárcamo, 2005).

El propósito del estudio fue conocer cuál es la percepción de esta estrategia en algunos establecimientos educativos que la han implementado, analizando los factores que puedan mejorar su impacto en los aprendizajes de los estudiantes. Considerando estos antecedentes, se planteó como objetivo general de la investigación develar la percepción sobre el trabajo colaborativo en la gestión curricular que tienen los profesores de educación regular y de educación especial/diferencial, que realizan a través de los Programas de Integración Escolar en escuelas básicas de la comuna de Tomé.

En consecuencia, el trabajo investigativo se desarrolló en torno a un conjunto de categorías y subcategorías deductivas de análisis, generadas a priori desde la teoría, las que fueron complementadas con categorías y subcategorías emergentes surgidas durante el análisis de los datos, que se presentan a continuación.

Tabla 1. Categorías y subcategorías de la investigación

\begin{tabular}{|c|c|}
\hline Categorías deductivas & Categorías emergentes \\
\hline $\begin{array}{l}\text { 1. Planificación de la enseñanza en el aula. } \\
\text { 1.2 Diseño de las adaptaciones curriculares. } \\
\text { 1.3 Preparación de recursos. } \\
\text { 1.4 Distribución de roles y responsabilidades. } \\
\text { 1.5 Determinación de las estrategias didácticas. }\end{array}$ & $\begin{array}{l}\text { 1.1 Planificación general del aula común. } \\
\text { 1.6 Planificación del enfoque de co-enseñanza. }\end{array}$ \\
\hline $\begin{array}{l}\text { 2. Didáctica de aula. } \\
\text { 2.1 Enfoque de co-enseñanza. } \\
\text { 2.2 Manejo de la conducta de los estudiantes. } \\
\text { 2.3 Comunicación entre los docentes. }\end{array}$ & $\begin{array}{l}\text { 2.4 Estrategias didácticas utilizadas. } \\
\text { 2.5 Relación entre actividades de aula común } \\
\text { y de aula de recursos. }\end{array}$ \\
\hline \multirow{2}{*}{$\begin{array}{l}\text { 3. Evaluación. } \\
\text { 3.1 y } 3.2 \text { Evaluación diagnóstica. } \\
\text { 3.4 Evaluación de proceso. } \\
\text { 3.5 Evaluación final. }\end{array}$} & $\begin{array}{l}\text { 3.1 Diagnóstico de los aprendizajes previos. } \\
\text { 3.2 Diagnóstico de las necesidades educativas } \\
\text { especiales. }\end{array}$ \\
\hline & $\begin{array}{l}\text { 4. Factores influyentes en el trabajo } \\
\text { colaborativo. }\end{array}$ \\
\hline
\end{tabular}

Fuente: elaboración propia.

\subsection{PARTICIPANTES}

Los sujetos fueron profesores(as) de escuelas básicas municipales de la comuna de Tomé. Se consideraron solamente escuelas básicas porque la mayoría de los estudiantes y docentes que participan en Programas de Integración Escolar pertenecen a este nivel educativo, y es donde la integración escolar lleva más tiempo funcionando. Los criterios de inclusión de los informantes claves se rigieron por las normas de ocupación de cargos docentes (MINEDUC, 2010). 
El muestreo se realizó de forma intencional, utilizando el tipo de casos desviantes o extremos para poder tener una muestra rica en información (Ruiz, 2007), considerando los años de experiencia, la formación de pregrado y el tipo de necesidad educativa especial con que trabajan los docentes. Se utilizó el criterio de saturación teórica para determinar la cantidad de sujetos entrevistados (Ruiz, 2007). En total, se incluyeron 12 sujetos, 6 profesores de educación regular y 6 de educación especial/diferencial. Respecto a las escuelas, se consideraron algunos indicadores generales como matrícula, nivel socioeconómico y puntaje promedio SIMCE.

\subsection{MÉTODO DE RELEVAMIENTO DE INFORMACIÓN}

Para obtener los datos se utilizó la técnica de entrevista semiestructurada, de modo de facilitar la profundidad de las respuestas y al mismo tiempo impedir la desviación de la conversación hacia puntos sin interés para la investigación (Baeza, 2002). Las entrevistas fueron realizadas de acuerdo a una guía de entrevista confeccionada con las categorías de investigación.

\subsection{PROCEDIMIENTOS}

Las entrevistas fueron realizadas con un aparato de registro sonoro para facilitar la construcción de los corpus. Además, se utilizó un registro de notas de campo para consignar aspectos no verbales, contextuales y situacionales.

En el análisis de los datos se utilizó el procedimiento de análisis sociológico del discurso en sus tres niveles de análisis: nivel textual, nivel contextual y nivel sociológico, ya que permite conjugar una serie de técnicas de análisis utilizando la lógica deductiva e inductiva, permitiendo caracterizar, comprender y explicar el discurso de los sujetos (Ruiz, 2009).

En la etapa de análisis de nivel textual de las entrevistas se realizó la transcripción del discurso oral de manera literal y detallada a partir de las grabaciones y las notas de campo. En particular, se utilizó la técnica de análisis de contenido, fragmentando y codificando el corpus en categorías apriorísticas y construyendo las categorías emergentes de la investigación. Luego se realizó un análisis descriptivo de tipo temático por cada categoría y subcategoría, modificando sus definiciones.

En la etapa de análisis de nivel contextual se consideró un análisis situacional e intertextual del discurso, en función de sus similitudes y diferencias con los discursos de otros informantes. Por otra parte, la etapa de análisis de nivel sociológico se realizó en su dimensión ideológica y como producto social.

Paralelamente, se construyeron memos durante el proceso de recogida y análisis de la información (Pérez, 2007), los que permitieron consignar y ordenar ideas para el análisis final.

\section{RESULTADOS}

\subsection{ANÁLISIS TEXTUAL Y CONTEXTUAL DE LA CATEGORÍA PLANIFICACIÓN DE LA ENSEÑANZA} EN EL AULA

Esta categoría corresponde a las actividades que realizan el profesor de educación regular y de educación especial/diferencial para planificar, preparar y organizar la ense- 
ñanza en el aula común.

En la subcategoría planificación general del aula común, los sujetos señalaron la existencia de un horario escaso para el trabajo colaborativo, debiendo utilizar tiempo informal por causa de problemas de contratación y coordinación de las horas entre los docentes. En relación a los temas abordados durante las reuniones de colaboración y la confección de las planificaciones, se detectó un rol primario del profesor de educación regular en su calidad de profesor jefe o profesor de asignatura, y un rol secundario y específico del profesor de educación especial.

Profesor de educación regular: No, si nos alcanzaba el tiempo, nos quedaba el tiempo y, es que tú ya vas manejando la idea y si tú tienes la disposición de repente tú estás en tu recreo, tomándote el café el de las nueve, nueve y media, y ahí: oye vamos a hacer esto aquí; apuntando y todo y tomándote un café tragándolo por decir y después te vienes a la sala, y trabajas $(09 / 158-162)^{1}$.

Entrevistador: En la preparación de tus clases del día a día, ¿tiene alguna injerencia ella, la profesora de educación especial?

Profesor de educación regular: No, no [...] (14/117-120).

En la subcategoría diseño de las adaptaciones curriculares, los docentes indicaron que éste es un ámbito específico de responsabilidad del profesor de educación especial. Los casos en que se relató un mayor grado de colaboración entre los docentes coinciden con los casos en que existen mayores grados de colaboración en la planificación del aula común, lo que se entiende como un intento de generar un mayor involucramiento del profesor de educación regular en el proceso educativo de estudiantes con necesidades educativas especiales.

Entrevistador: ¿Cómo es la colaboración con la profesora regular para hacer esas adaptaciones?

Profesor de educación especial: Bueno es, hay diferentes, o sea así hablando sinceramente hay diferentes, porque hay algunas de ellos que aceptan esto, aceptan que adecuemos que, nos apoyan, pero hay otros que, que no están tan convencidos de esto entonces, es más difícil, hay que buscar ahí la forma de llegar para que se cumpla (12/26-29).

En la subcategoría preparación de recursos se detectó un rol principal del profesor de educación regular, mientras se observó un rol secundario y complementario del profesor de educación especial, a quien se le señaló que no tiene participación en la planificación del resto del proceso educativo. Cabe mencionar que algunos sujetos indicaron que efectivamente realizaban un trabajo conjunto, sin embargo, se trata de docentes que además mantienen vínculos personales de amistad.

Profesor de educación regular: [...] [la profesora diferencial de alumnos con necesidades educativas especiales permanentes] me ayudaba a preparar material

El paréntesis indica: el número de entrevista/las líneas del corpus. 
concreto por ejemplo para los niños con necesidad, por ejemplo cuando trabajábamos fracciones, ya había que llevar tal material vamos a trabajar esto, ella [llevaba] material concreto para los niños con necesidad, [...] entonces ella preparaba el material [...] (3/29-32).

En la subcategoría distribución de roles y responsabilidades, hay una amplia convergencia en que no existe un acuerdo previo de distribución de roles y responsabilidades entre los docentes, sino que se asume una distribución caracterizada por el rol principal del profesor de educación regular, especialmente en su calidad de profesor jefe del curso y un rol secundario por parte del profesor de educación especial.

Entrevistador: ¿Existe alguna determinación o se asume una distribución?

Profesor de educación especial: Se asume más que se diga lo que tú tienes que hacer, sino que se asume a la larga el proceso [...] (11/86-88).

En la subcategoría planificación del enfoque de co-enseñanza, en su mayoría se detectó tanto la ausencia de una distribución previa de las actividades docentes en aula común, como un desconocimiento de los enfoques de co-enseñanza. Los sujetos que relataron la existencia de una distribución de las actividades de aula, describieron modelos de trabajo que se ajustan al rol principal del profesor de educación regular en el aula común y el rol secundario, complementario y de apoyo del profesor de educación especial. Los casos excepcionales de acercamiento al modelo de co-enseñanza en equipo se relacionan con situaciones en que los docentes de educación especial han logrado una mayor injerencia en los ámbitos de la planificación, pero circunscritos a las clases que ambos docentes comparten, y centrados en los estudiantes del Programa de Integración Escolar.

Entrevistador: Esas intervenciones [de la profesora diferencial en la clase], ¿están puestas de acuerdo desde antes o surgen en el momento?

Profesor de educación especial: Surgen en el momento, no, surgen en el momento (12/183-184).

Profesor de educación especial: Es que claro porque yo siempre le explico antes a la profesora cuál es cómo es la forma en que yo voy a trabajar con ella.

Entrevistador: Tú siempre le has explicado.

Profesor de educación especial: Sí, de hecho siempre quedamos de acuerdo en lo mismo [el profesor regular dirige la clase y el profesor diferencial trabaja con los alumnos individualmente y realiza intervenciones complementarias] (10/147-152).

\subsection{ANÁLISIS TEXTUAL Y CONTEXTUAL DE LA CATEGORÍA DIDÁCTICA DE AULA}

Esta categoría corresponde a las actividades de enseñanza que realizan colaborativamente el profesor de educación regular y de educación especial/diferencial en el aula común.

En la subcategoría enfoque de co-enseñanza, la mayoría de los docentes describieron los modelos de co-enseñanza de apoyo y complementaria, con una menor cantidad de referencias al enfoque de co-enseñanza de rotación entre grupos y acercamientos a los enfoques de grupos simultáneos, de observación y en equipo. Además, se debe notar el uso 
de la palabra "intervención” para nombrar las acciones que realiza el profesor de educación especial en el aula regular. Esto se relaciona con el rol preponderante que tiene el profesor de educación regular dentro del aula común, frente a un rol secundario, complementario y más específico del profesor de educación especial.

Entrevistador: Y por ejemplo cuando tú dirigías la clase, ¿qué hacía la otra profesora?

Profesor de educación regular: ¿Qué hacía la Andrea [profesora de educación especial]? Trabajaba con su grupo, con los chicos que tenía o de repente apoyando dentro del curso en general [...] (3/160-162).

Profesor de educación especial: A ver, con los [profesores regulares] que te permiten la intervención tú puedes aportar con ejemplos, de repente a lo mejor la clase no está tan coordinada contigo pero en el momento tú vas aportándole ejemplos, le puedes hacer, que el aprendizaje que ellos, llevarlo a la vida cotidiana de los niños, para qué les va a servir [...] (11/128-130).

En la subcategoría manejo de la conducta de los estudiantes, la mayoría de los casos indicaron que este poder se concentra en el profesor de educación regular, mientras que el profesor de educación especial es un elemento de consulta y asesoramiento. También, cuando los profesores diferenciales determinaban las estrategias y normas para los estudiantes con necesidades educativas especiales asociadas a dificultades conductuales, se reitera el esquema anterior, pues se entrega la responsabilidad de casos específicos a los docentes que se consideran especialistas en el área. Solamente en un caso, en que los docentes relataron compartir y colaborar en la determinación, se observa un equilibrio de poder y de roles. Este último caso está relacionado con las demás subcategorías en que se trata de docentes que indicaron desarrollar un mayor grado de colaboración en la planificación y tener relaciones interpersonales de afinidad y cercanía favorables al trabajo colaborativo.

Entrevistador: ¿Pero quién determina cómo se van a tratar las conductas de los estudiantes?

Profesor de educación especial: Ah, quién determina, ya, depende, porque si el caso por ejemplo el diagnóstico de mi alumno está cien por ciento ligado a las conductas que él presenta dentro del aula, soy yo la que determina cómo se trabaja, pero si el diagnóstico que él tiene no se asocia al tipo de conductas que él tiene, eso lo determina la profesora, obviamente (10/162-165).

En la subcategoría comunicación entre los docentes, se detectó la ausencia de determinación de estrategias de comunicación entre los docentes durante el desarrollo de las clases, por lo que éstas han surgido de manera espontánea durante la práctica. Esto indica que no existe una planificación y organización de la co-enseñanza.

Profesor de educación especial: Sabes que fue espontáneo, no fue como ya sabes que, cuando queramos hablarnos te espero en la esquina de la sala, no, es como espontáneo, porque así surgió (2/168-171). 
En la subcategoría estrategias didácticas utilizadas, cabe destacar que tres cuartas partes de las citas de esta subcategoría (27 de 36) corresponden a profesores de educación regular, lo cual es un indicio del rol preponderante que tienen ellos durante el desarrollo de las clases.

En la subcategoría relación entre actividades de aula común y de aula de recursos, algunos sujetos indicaron la existencia de una vinculación entre los aprendizajes y habilidades trabajadas en el aula común y el aula de recursos. Con los estudiantes que están al mismo nivel curricular del curso, se trabaja en aula de recursos en forma paralela al aula común, retomando los contenidos y reforzando, pero cuando los estudiantes tienen un desfase curricular mayor se trabajan aprendizajes de un nivel inferior.

Profesor de educación especial: En ocasiones, depende del niño, si los niños están a nivel del curso o el aprendizaje de esa unidad está planteado en los mismos que sus compañeros, es fácil de vincularlo, se va trabajando a la par que el colega, pero cuando los niños, hay un desfase del currículum, uno trabaja las competencias inferiores para ir logrando aprendizajes con ellos (11/289-294).

\subsection{ANÁLISIS TEXTUAL Y CONTEXTUAL DE LA CATEGORÍA EVALUACIÓN}

Corresponde a las actividades que realizan el profesor de educación regular y de educación especial/diferencial para evaluar, monitorear y retroalimentar el proceso de enseñanza-aprendizaje de los estudiantes en el contexto de aula común, especialmente de aquéllos con necesidades educativas especiales.

En la subcategoría diagnóstico de los aprendizajes previos, los docentes relataron que el diseño y la aplicación de los instrumentos diagnósticos estaba a cargo del profesor de educación regular. Los casos en que las evaluaciones diagnósticas se analizaban en forma colaborativa coinciden con un mayor grado de colaboración en la planificación y en la didáctica, tratándose de docentes que mantienen vínculos interpersonales.

Profesor de educación especial: Bueno, la profesora jefe prepara las evaluaciones, prepara las evaluaciones diagnósticas, de todos los niños, con excepción obviamente de los permanentes, de los niños permanentes, porque ellos están en un nivel más bajo que el curso, entonces ahí pide que uno prepare el instrumento, la evaluación diagnóstica, pero con los transitorios se trabaja igual, la misma evaluación diagnóstica (12/201-205).

En cambio, en la subcategoría diagnóstico de las necesidades educativas especiales se relató un escaso involucramiento del profesor de educación regular en el proceso, que junto al carácter informativo de la entrega de resultados de la evaluación a los profesores regulares, indican que el ámbito de diagnóstico de las necesidades educativas especiales es una responsabilidad preferente de los profesores de educación especial, desarrollándose un escaso trabajo interdisciplinario.

Profesor de educación especial: Bueno, nosotros al profesor [de educación regular] le informamos, cierto, después de hacer la evaluación pertinente al alumno, le informamos cuál fue el resultado [...] si el chico necesita el apoyo o no 
necesario y se le da a conocer tanto a él como al jefe de UTP los resultados de la evaluación, y ver cuál va a ser la forma que lo vamos a ayudar (1/336-340).

En la subcategoría evaluación de proceso, los docentes señalaron que las evaluaciones eran realizadas principalmente por el profesor de educación regular, con injerencia del profesor diferencial en algunos casos, quien orientaba la confección de los instrumentos para estudiantes con necesidades educativas especiales. También en algunos casos los instrumentos eran elaborados por los profesores diferenciales.

Profesor de educación especial: La prueba la hace él, absolutamente, después el profesor conversamos, viene para acá cierto, conversamos, vemos sabes que yo encuentro que para mis alumnos tú estás muy elevado, bajémosle el nivel y arreglamos la prueba especialmente para el alumno (2/268-270).

En la subcategoría evaluación final, los sujetos convergieron en que existe una determinación conjunta de la promoción de los estudiantes con necesidades educativas especiales. En los casos divergentes, ocurre que los profesores de educación especial toman la decisión de promoción de los estudiantes, señalando así el rol más específico del profesor de educación especial.

Entrevistador: Y en la promoción a fin de año.

Profesor de educación especial: $\mathrm{Si}$, ahí hay incidencia, si ahí hay incidencia, hay conversaciones previas, se busca el espacio, el tiempo y se genera esto de poder establecer qué tan beneficioso o que, qué tan perjudicial sería una repitencia para el niño según el estado cognitivo, el estado pedagógico, el estado emocional, sí, ahí sí, y cuando finalmente se llega a un acuerdo se expone en UTP, y se exponen como te digo, las razones, se fundamenta bien porque si no, no se produce, claro porque no es repetir por repetir.

Entrevistador: ¿Con todos los profesores lograste hacer eso?

Profesor de educación especial: Sí, no, con todos [...] (5/613-620).

En las escuelas que realizan instrumentos de evaluación final, algunos docentes señalaron no adaptarlas a los estudiantes con necesidades educativas especiales, lo que indica el fuerte peso que tienen las políticas generales de la escuela sobre las necesidades y características reales de los estudiantes. En aquellos casos en que existe algún tipo de adaptación de los instrumentos, se realizaba en forma conjunta entre los docentes, lo que indica un espacio en donde se equilibran las funciones de los docentes.

\subsection{ANÁLISIS TEXTUAL Y CONTEXTUAL CATEGORÍA FACTORES INFLUYENTES EN EL TRABAJO COLABORATIVO}

Corresponde a los factores que influyen y explican la forma particular en que se desarrolla el trabajo colaborativo.

Los discursos se desarrollaron en torno a ámbitos como la disponibilidad de tiempo, las políticas y orientaciones técnicas, el rol de los directivos, el funcionamiento de los procesos técnicos, las relaciones interpersonales, la motivación individual, la didáctica 
docente, la formación profesional y la edad. De ellos, los factores más recurrentes y transversales en el discurso de los sujetos fueron la escasa disponibilidad de tiempo para realizar trabajo colaborativo, el rol de la gestión de los directores y jefes técnicos y los aspectos interpersonales.

Profesor de educación especial: Tiempo, tiempo, si ese es el mayor problema digamos, tiempo, hace falta mucho tiempo porque uno no alcanza de repente, de repente por ejemplo tenemos trabajo colaborativo y vienen apoderados, entonces ya hay que dejar el tiempo porque no hay espacio para atender apoderados, no hay espacio para hacer planificaciones, para hacer, al final uno lleva el trabajo para la casa, porque no hay tiempo acá en la escuela para hacer guías, entonces el factor tiempo es importante, ése yo creo que es nuestro peor, es lo más malo que nos puede pasar a todos, tiempo para el trabajo colaborativo (12/280-286).

Profesor de educación especial: [...] y habían otros [profesores regulares] que yo creo que por una mala gestión de la dirección de la escuela, y también por, por una despreocupación y falta de, falta de compromiso, se les designaron otras labores sabiendo que ellos tenían esas horas de colaboración, entonces por ejemplo habían profesores donde tú les buscabas y ellos te decían: a mi me gustaría estar contigo pero en realidad me mandaron a hacer reforzamiento a matemática; justo el día y a la hora en que estabas ahí [...] (5/218-222).

Profesor de educación regular: Mira, se da por, yo creo que por tres razones, más o menos, mira, la primera es porque con la Andrea [profesora de educación diferencial], la Andrea es una persona que te escucha, o sea ella te escucha, ella da lugar a que alguien más le diga cosas que tiene que hacer, ponte tú no sé: ya Andrea haz esto, y ella lo va a hacer, ella no es dueña de sus propias ideas, no sé si me entiendes (4/88-91).

Dos de ellos indican cómo los sujetos atribuyeron las características del trabajo colaborativo a factores externos que dificultan su realización, mientras el otro es un factor interno de las personas que afecta positivamente el desarrollo del trabajo colaborativo.

\subsection{ANÁLISIS SOCIOLÓGICO}

$\mathrm{Al}$ analizar los discursos desde una perspectiva ideológica, se evidencia el peso que tiene la cultura escolar, entendida como "el conjunto de prácticas y simbolizaciones construidas en la escuela" (Redón, 2011: 450) sobre la forma en que se desarrolla el trabajo colaborativo.

Se observa la persistencia de una concepción de la educación especial marcada por el modelo médico-rehabilitador (Arnaiz, 2000), en aspectos como la centralidad del diagnóstico y su influencia sobre las adaptaciones curriculares, la exclusión del uso de estrategias de adaptación curricular a los estudiantes que no están incorporados al Programa de Integración Escolar, la consideración de la educación especial como un ámbito preferente de profesionales especializados, y el modelo de consulta bajo el cual actúa el profesor de educación especial durante el proceso de diagnóstico de las necesidades educativas especiales y durante la determinación de las normas de aula común. 
También se observa la influencia que tiene la cultura de trabajo pedagógico aislado de la docencia tradicional (Waldron \& Mcleskey, 2010), en donde cada profesor se hace cargo de su clase en forma individual. De este modo, el profesor de educación regular sigue siendo el principal responsable de la gestión curricular del aula común, dificultando el desarrollo de una auténtica co-enseñanza entre los docentes.

Un aspecto relevante dentro de la cultura escolar es la persistencia de una concepción separada (discriminadora) de la educación regular y la educación especial, en donde esta última tiene un rol secundario y especializado al interior de las escuelas regulares. Esto se evidencia en el rol principal que tiene el profesor de educación regular dentro de la gestión curricular del aula común, en aspectos como la planificación de aula común, la determinación y la elaboración de los recursos didácticos, la distribución de roles y responsabilidades, la determinación de las estrategias didácticas, el manejo de la conducta de los estudiantes, los modelos de co-enseñanza utilizados, el diseño de procedimientos e instrumentos de evaluación, y la escasa transferencia de elementos metodológicos de la educación especial a los estudiantes del curso que no pertenecen al Programa de Integración Escolar. En cambio, el profesor de educación especial ejerce un rol de apoyo en la gestión curricular del aula común.

No obstante, el profesor de educación especial tiene un gran protagonismo en la elaboración de las adaptaciones curriculares y en la evaluación diagnóstica de las necesidades educativas especiales, lo que es concordante con el rol especializado que se le atribuye a la educación especial y la predominancia de un enfoque médico-rehabilitador. Por otro lado, el carácter compartido entre los docentes de la promoción de los estudiantes con necesidades educativas especiales puede deberse a la importancia e impacto de la promoción en los estudiantes, por lo que ningún docente se siente habilitado para tomar tal decisión por sí mismo.

Considerando un análisis del discurso como producto social, se debe notar que la atribución a factores externos de las dificultades para realizar el trabajo colaborativo, junto con la atribución a factores internos del desarrollo de un mejor trabajo colaborativo, es coincidente con la teoría de la atribución de Robbins (1999).

También se encontró discrepancia entre las políticas de la escuela regular y el funcionamiento del Programa de Integración Escolar, evidenciada en las dificultades de coordinación entre los docentes y la predominancia de las políticas generales de la escuela regular por sobre las necesidades individuales de los estudiantes en la gestión curricular.

Teniendo en cuenta el concepto de cohesión social entendido como el sentido de pertenencia, conexión y visión común entre los actores sociales (Moiij, Smeets \& de Wit 2011), se observa que el trabajo colaborativo se ve dificultado por un problema de cohesión a nivel de la cultura y de la organización escolar, consistente en una separación y, probablemente, un conflicto de ámbitos de competencia entre la educación regular y la educación especial, y un conflicto entre los docentes y la organización educativa. Este conflicto entre los docentes y la organización educativa posiblemente está enmarcado en un conflicto de resistencia entre los profesores y las exigencias cada vez mayores de la gestión escolar.

Cabe destacar la influencia que los sujetos atribuyeron a los vínculos sociales interpersonales en el trabajo colaborativo, indicando que cuando existen fuertes vínculos personales entre los docentes se genera cohesión, lo que permite el desarrollo de mayores grados de colaboración, a pesar de las barreras existentes a nivel organizacional e institucional. 
De lo anterior, se desprende que la escuela en su globalidad (sus políticas, normativas, forma de organización y cultura escolar) produce un discurso focalizado en el trabajo individual más que social (o de trabajo en equipo), lo que favorece el surgimiento de conflictos de competencia, separación de las responsabilidades, escasa integración profesional, poco enriquecimiento interdisciplinario, y dificulta el alineamiento de los docentes con un proyecto educativo, curricular y didáctico común, que incluya y coordine de forma constructiva a todos los profesionales que laboran en el establecimiento para el aprendizaje de todos los estudiantes, que es en definitiva el objetivo central de la educación, especialmente desde una perspectiva inclusiva.

En nuestra opinión, la idea de una comunidad de aprendizaje de carácter inclusivo se encuentra lejos de ser una realidad en estos contextos, a pesar de las múltiples voces a su favor dentro y fuera de las escuelas, pues existen múltiples factores organizacionales y de formación que dificultan su realización. Se infiere de los sujetos la existencia de una paradoja educacional caracterizada por la exigencia y el deseo de transformar la educación hacia nuevas formas de trabajo que buscan mejorar la calidad de los aprendizajes, pero con aspectos organizacionales y culturales que frenan bruscamente la realización de estos cambios.

Figura 1. Diagrama que explica la influencia de los discursos de los sujetos respecto a la escasa práctica de trabajo colaborativo

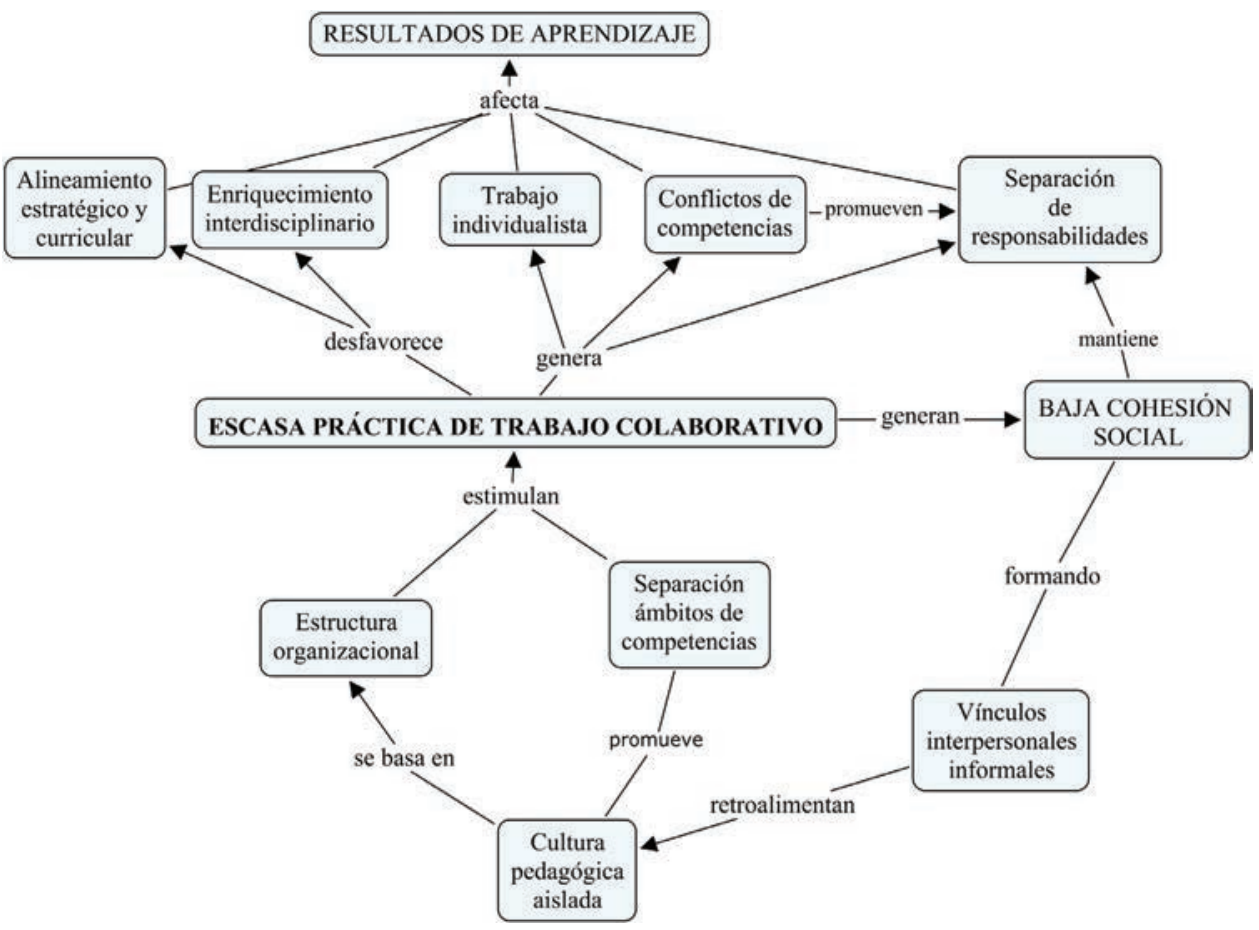

Fuente: elaboración propia. 


\section{CONCLUSIONES}

Este estudio ha permitido tener una acercamiento empírico a la forma en que se está desarrollando el trabajo colaborativo entre los docentes, y entrega una base para plantear recomendaciones para el diseño e implementación de esta política educativa, junto con líneas de investigación futuras respecto al tema.

Desde el análisis textual se puede observar que los sujetos perciben una realidad que va desde un continuo de escaso trabajo colaborativo, limitado a una relación de consulta entre los docentes, a un trabajo colaborativo más desarrollado caracterizado por una coenseñanza incipiente o inicial. Dentro de ello destacamos el acercamiento de los docentes a algunos enfoques de co-enseñanza descritos en la literatura, como la co-enseñanza de grupos simultáneos y en equipo, sin haber tenido la formación y orientación apropiadas al respecto. Coincide con la literatura el uso predominante del enfoque de apoyo y de rotación entre grupos en docentes que se inician en la co-enseñanza (Scruggs, Mastropieri \& McDuffie, 2007; Villa, Thousand \& Nevin, 2008).

Respecto al uso del enfoque de apoyo, ocurre que generalmente el rol de dirigir la clase recae en el profesor regular, lo que favorece la percepción del profesor de educación especial como secundario frente al profesor regular, coincidiendo con lo planteado por Hughes \& Murawski (2001). Este rol secundario se observa en la mayoría de las subcategorías estudiadas, como la planificación del aula común, la determinación de las estrategias y recursos didácticos, el manejo de la conducta, la evaluación de los aprendizajes previos y la evaluación de proceso. En cambio, los sujetos relataron un rol mayor del profesor de educación especial en aspectos tradicionalmente considerados como propios de la educación diferencial, correspondientes a las subcategorías de elaboración de las adaptaciones curriculares y evaluación diagnóstica de las necesidades educativas especiales. En conjunto, esto evidencia un rol del profesor de educación especial más cercano a un docente de apoyo que a un co-educador, lo que coincide con los hallazgos de investigaciones similares llevadas a cabo en otros países (Friend et al., 2010).

Del análisis contextual destacamos los vínculos entre algunas categorías respecto a los diferentes grados de colaboración, por ejemplo, cuando los sujetos perciben un mayor grado de colaboración en la planificación suelen hacerlo también en la didáctica, aunque no ocurre lo mismo en todas las subcategorías de la evaluación. Del mismo modo, los sujetos no describieron una distribución explícita de roles y responsabilidades ni de comunicación entre los docentes, incluso entre aquellos que indicaron desarrollar un mayor trabajo colaborativo. Esto indica que el trabajo colaborativo se ha realizado de forma improvisada, sin una planificación de la co-enseñanza, focalizando la colaboración en algunos ámbitos de la gestión curricular y no en su globalidad. La planificación es fundamental para una práctica adecuada de esta estrategia, por lo cual quienes gestionan su implementación, tanto en el Ministerio de Educación como en las escuelas, deben garantizar la existencia de espacios para planificar la co-enseñanza, evitando el desarrollo improvisado que ha tenido hasta ahora.

Del análisis sociológico resaltamos los elementos culturales y sociales que se han podido desprender desde la percepción de los sujetos. La persistencia de concepciones culturales desfavorables para la co-enseñanza, como el enfoque médico-rehabilitador de la educación especial, la visión separada entre la educación especial y regular y la cultura de trabajo pedagógico aislado, y por otro lado, el hallazgo de un problema de cohesión social al interior de las escuelas, indican que estos aspectos deben considerarse al momento de 
diseñar e implementar políticas públicas en educación, de manera de promover no sólo cambios en las prácticas, sino también en la cultura escolar y las relaciones sociales que se desarrollan en las escuelas. Algunos de estos aspectos culturales habían sido detectados en estudios anteriores sobre la integración escolar en Chile, aunque sin ahondar en los aspectos más sociológicos (Castro y Figueroa, 2006; CEAS, 2004).

En conjunto, esto indica que la co-enseñanza es una estrategia posible de llevar a cabo en nuestra realidad, pero que requiere que las escuelas cambien su tendencia a favorecer un sistema de trabajo atomizado en vez de un trabajo colaborativo y en equipo. Esto implica que debe ser considerada la presencia de las barreras organizacionales, culturales y sociales halladas en esta investigación. También, entre las barreras para la co-enseñanza hay que agregar la sobrecarga de trabajo de los docentes (Stuart et al., 2006), las actitudes y creencias personales desfavorables a la colaboración (Araneda et al., 2008; Castro y Figueroa, 2006; Stuart et al., 2006), y la falta de estrategias para resolver problemas en forma colaborativa entre los docentes (Sánchez, 2000). De manera especial se deben potenciar aquellos elementos facilitadores del trabajo colaborativo, partiendo por un mayor apoyo organizacional en cuanto a disponibilidad de tiempo, coordinación y orientación (Murawski \& Dieker, 2004; Villa, Thousand \& Nevin, 2008), una formación profesional explícita sobre el tema, la presencia de actitudes personales favorables al trabajo con otros profesionales, la creación de una relación de confianza, horizontalidad y compromiso entre los docentes (Graden y Bauer, 1999; Murawski \& Dieker; 2004, Villa, Thousand \& Nevin, 2008), y en general el fomento de una cultura colaborativa (Waldron \& Mcleskey, 2010).

Otros aspectos que deben ser investigados son, por un lado, la influencia que ejerce la cultura escolar en el éxito o fracaso de nuevas prácticas educativas, considerando de qué manera la transformación en las prácticas educativas interactúa con las transformaciones en la cultura escolar. En relación a ello, se debiera profundizar en la problemática de la cohesión social en las escuelas, para ahondar en el peso que ejerce sobre el desarrollo de las prácticas educativas. Por otro lado, retomando a Cramer et al. (2010), se confirma la necesidad de revisar la formación actual de profesores en virtud de la necesidad de incrementar la colaboración entre profesionales, el trabajo interdisciplinario y especialmente la co-enseñanza.

Así mismo, se deben considerar las limitaciones de este estudio. En primer lugar, se deben atender las limitaciones propias de un estudio cualitativo en términos de representatividad de la muestra y generalización de los resultados. Una segunda limitación tiene que ver con la elección del tipo de estudio de naturaleza descriptiva, y la dificultad para construir una teoría explicativa. Para ello sería interesante diseñar un estudio explicativo sobre los factores influyentes en el trabajo colaborativo.

Un elemento que no fue abordado durante la investigación, pero que urge en el diseño y aplicación de esta estrategia, es el grado de voluntariedad de la co-enseñanza. Autores como Graden y Bauer (1999) y Murawski \& Dieker (2004) han señalado la importancia de que la enseñanza colaborativa sea voluntaria. Por otro lado, varias investigaciones recogidas por Scruggs, Mastropieri \& McDuffie (2007) evidencian que cuando la co-enseñanza es voluntaria se desarrolla de forma más exitosa, en cambio cuando es forzada aumenta el fracaso. Incluso en los casos donde los docentes están de acuerdo con la obligatoriedad de la co-enseñanza, se recomienda "que debe ser graduada durante un periodo de años y acompañada por una formación y apoyo suficiente" (Scruggs, Mastropieri \& McDuffie, 2007: 404). 


\section{REFERENCIAS BIBLIOGRÁFICAS}

Araneda, P., Frei, E., González, F., Millán, L., Rey, V., Tenorio, S., Yñesta, A. y Flores, F. (2008). Estudio de la calidad de la Integración Escolar. Recuperado de http://www.mineduc.cl/ usuarios/edu.especial/File/DOCUMENTOS\%202010/ESTUDIOS\%20Y\%20DOCUMENTOS/ EstudioIntegraciOnEscolarUMCE.pdf, consultado en septiembre de 2011.

Arnaiz, P. (2000). Educar en y para la diversidad. En F. Soto y J. López (Eds.), Nuevas tecnologías, viejas esperanzas: Las nuevas tecnologías en el ámbito de las necesidades especiales y la discapacidad (pp. 29-40). Murcia: Consejería de Educación y Universidades.

Baeza, M. (2002). De las metodologías cualitativas en investigación científico social. Concepción: Universidad de Concepción.

Beamish, W., Bryer, F. \& Davies, M. (2006). Teacher Reflections on Co-teaching a Unit of Work. International Journal of Whole Schooling, vol. 2, n. 2, 3-18.

Cárcamo, H. (2005). Hermenéutica y análisis cualitativo. Cinta de Moebio, n. 23. Recuperado de http://www2.facso.uchile.cl/publicaciones/moebio/23/carcamo.htm, consultado en noviembre de 2011.

Castro, R. y Figueroa, B. (2006). Trabajo colaborativo en escuelas especiales de lenguaje, un enfoque educativo curricular. Paideia, n. 41, 117-127.

Consultora en Estudios, Asesorías y Planificación en Desarrollo Local (CEAS). (2004). Estudio a nivel muestral de la calidad del proceso de integración educativa. Recuperado de http:// www.mineduc.cl/usuarios/edu.especial/File/DOCUMENTOS\%202010/ESTUDIOS\%20Y\%20 DOCUMENTOS/EstudioIntegraciOnCEAS.pdf, consultado en septiembre de 2011.

Cramer, E., Liston, A., Nervin, A. \& Thousand, J. (2010). Co-teaching in Urban Secondary School. Districts to Meet the Needs of all Teachers and Learners: Implications for Teacher Education Reform. International Journal of Whole Schooling, vol. 6, n. 2, 59-76.

Friend, M., Cook, L., Hurley-Chamberlain, D. \& Shamberger, C. (2010). Co-Teaching: An Illustration of the Complexity of Collaboration in Special Education. Journal of Educational and Psychological Consultation, vol. 20, n. 1, 9-27.

Graden, L. y Bauer, M. (1999). Enfoque colaborativo para apoyar al alumnado y profesorado de aulas inclusivas. En S. Stainback y W. Stainback (Eds.), Aulas inclusivas (pp. 103-117). Madrid: Narcea.

Hughes, C. \& Murawski, W. (2001). Lessons from another Field: Applying Co-teaching Strategies to Gifted Education. Gifted Child Quarterly, vol. 45, n. 3, 195-203.

MINEDUC. (2012a). Orientaciones actualizadas para la implementación del Decreto Supremo 170/2009 en Programas de Integración Escolar. Capítulo III. Recuperado de http://www.mineduc. cl/usuarios/edu.especial/doc/201209121852110.CAPITULOIII.pdf, consultado en marzo de 2012.

MINEDUC. (2012b). Orientaciones técnicas para Programas de Integración Escolar. Recuperado de http://www.educacionespecial.mineduc.cl/usuarios/edu.especial/File/2012/ SEPTIEMBRE2012/OrientTecPIEWEB.PDF, consultado en septiembre de 2012.

MINEDUC. (2010). Orientaciones para la implementación del Decreto $N^{o} 170$ en Programas de Integración Escolar. Recuperado de http://www.mineduc.cl/usuarios/edu.especial/ doc/201104141100390.ORIENTACIONES_IMPLEMENTACION_DTO170_PIE_v2.pdf, consultado en septiembre de 2011.

MINEDUC. (2009). Decreto Supremo 170 de 2009 que fija normas para determinar los alumnos con necesidades educativas especiales que serán beneficiados de las subvenciones para educación especial. Recuperado de http://www.mineduc.cl/biblio/documento/201005031126500. DEC200900170.pdf, consultado en octubre de 2010.

MINEDUC. (2008). Orientaciones para dar respuestas educativas a la diversidad y a las necesidades educativas especiales. Recuperado de http://www.mineduc.cl/usuarios/edu.especial/ doc/201105102232260.ORIENTACIONES_RESPUESTAS_A_LA_DIVERSIDAD.pdf, consultado en octubre de 2010 . 
Moiij, T., Smeets, E. y de Wit, W. (2011). Multi-level Aspects of Social Cohesion of Secondary Schools and Pupils' Feelings of Safety. British Journal of Educational Psychology, vol. 81, n. 3, 369-390.

Moliner, O. (2008). Condiciones, procesos y circunstancias que permiten avanzar hacia la inclusión educativa: Retomando las aportaciones de la experiencia canadiense. Revista Latinoamericana de Educación Inclusiva, vol. 2, n. 1, 41-60.

Murawski, W. (2008). Five Keys to Co-teaching in Inclusive Classrooms. School Administrator, vol. 65, n. $8,29$.

Murawski, W. \& Dieker, L. (2004). Tips and Strategies for Co-teaching at the Secondary Level. Teaching Exceptional Children, vol. 36, n. 5, 52-58.

Pérez, G. (2007). Investigación cualitativa. Retos e interrogantes. Madrid: La Muralla.

Redón S. (2011). Escuela e identidad: Un desafío docente para la cohesión social. Polis, vol. 10, n. $30,447-476$.

Robbins, S. (1999). Comportamiento organizacional. México D.F.: Prentice Hall.

Ruiz Olabuénaga, J. (2007). Metodología de la investigación cualitativa. Bilbao: Universidad de Deusto.

Ruiz Ruiz, J. (2009). Análisis sociológico del discurso: Métodos y lógicas. Forum: Qualitative Social Research, vol. 10, n. 2. Recuperado de http://nbn-resolving.de/urn:nbn:de:0114-fqs0902263, consultado en enero de 2012.

Sánchez, E. (2000). El asesoramiento psicopedagógico: Un estudio observacional sobre las dificultades de los psicopedagogos para trabajar con los profesores. Infancia y aprendizaje, n. 91, 55-77.

Scruggs, T., Mastropieri, M. y McDuffie, K. (2007). Co-teaching in Inclusive Classrooms: A Metasynthesis of Qualitative Research. Exceptional Children, vol. 73, n. 4, 392-416.

Stainback, S. y Stainback, W. (1999). Aulas inclusivas. Madrid: Narcea.

Stuart, S., Connor, M., Cady, K. \& Zweifel, A. (2006). Multiage Instruction and Inclusion: A Collaborative Approach. International Journal of Whole Schooling, vol. 3, n. 1, 12-23.

Villa, R., Thousand, J. \& Nevin, A. (2008). A Guide to Co-teaching. Practical Tips for Facilitating Student Learning. Thousand Oaks: Corwin Press.

Waldron, N. \& Mcleskey, J. (2010). Establishing a Collaborative School Culture through Comprehensive School Reform. Journal of Educational and Psychological Consultation, vol. 20, n. $1,58-74$. 
